\title{
Is there A Role for Expanding the Indication for TEVAR in Acute Type B Aortic Dissections? A Further Classification of Uncomplicated Dissections
}

\author{
Alex Sher and Rami O Tadros
}

Mount Sinai Medical Center, Department of Vascular Surgery, USA

*Corresponding author: Rami O Tadros, Mount Sinai Medical Center, Department of Vascular Surgery, USA, Tel: 12122415393; E-mail: rami.tadros@mountsinai.org

Received date: July 15, 2016; Accepted date: July 26, 2016; Published date: August 02, 2016

Copyright: (c) 2016 Sher A, et al. This is an open-access article distributed under the terms of the Creative Commons Attribution License, which permits unrestricted use, distribution, and reproduction in any medium, provided the original author and source are credited.

Citation:Sher A, Tadros RO. Is there a Role for Expanding the Indication for TEVAR in Acute Type B Aortic Dissections? A Further Classification of Uncomplicated Dissections. J Vasc Endovasc Surg. 2016, 1: 16.

\section{Background}

The treatment of Stanford type B (DeBakey III) aortic dissections has been evolving, as evidenced by over a dozen studies published in the past few months. Historically, management has consisted of medical therapies and long-term surveillance with the ultimate goal of limiting the impulse force on the aorta [1]. A quarter of patients with type B aortic dissections present with accompanying rupture, aneurysmal degeneration, aortic dilatation, malperfusion, and/or refractory pain and are considered complicated [2,3]. These patients often warrant aggressive and immediate treatment with surgery, and more recently thoracic endovascular aortic repair (TEVAR), as failure to treat is associated with severe complications (e.g., organ damage and death). In fact, many expert consensus statements recommend TEVAR as the initial and a potentially lifesaving therapy for acute complicated type $B$ dissections [4]. The other three-quarters of patients are traditionally classified as uncomplicated and conservatively managed with antihypertensive and pain medications alone. However, a large proportion of these patients end up undergoing secondary intervention, and experience severe complications or even death [5-11]. With 30\% cumulative mortality at 5 years and less than 50\% long-term survival [12], many surgeons are beginning to question if the indications for TEVAR should be expanded to treat certain uncomplicated type $B$ aortic dissections.

\section{Evidence for expanding TEVAR}

To date, no level I evidence supporting the use of TEVAR for uncomplicated type B aortic dissections exists. The lack of randomized controlled trials on the subject and advancements in endovascular therapies has allowed for some deviation from the customarily high indications required to intervene. The recent U.S. Food and Drug Administration's approval of endovascular devices for the treatment of uncomplicated type $B$ dissections is evidence of this movement.

Despite its growing use, limited data exists on the results comparing TEVAR and best medial therapy to medical therapy alone in the population initially diagnosed uncomplicated. The
INSTEAD-XL (Investigation of Stent Grafts in Patients with Type B Aortic Dissection) randomized trial offers recent long-term data suggesting prophylactic TEVAR in addition to medical management has superior outcomes for this group [12]. In this study, all-cause mortality, aorta-specific mortality, and freedom from progression of disease or aortic-specific event all favored TEVAR plus medical therapy. Another multicenter randomized controlled trial, ABSORB (Acute Dissection Stent grafting or Best medical treatment) found similar results [13]. Both showed that medical therapy alone failed to achieve the favorable remodeling of the true/false lumen and false lumen thrombosis seen after TEVAR.

Some studies that have countered early TEVAR cite one finding in the INSTEAD trial that showed failure to improve survival of patients in less than 2 years of follow-up. However, progression of disease after 2 years was worse for the group treated with medical therapy alone. This suggests that the benefits of TEVAR (aortic remodeling, reperfusion of true lumen, thrombosis of false lumen) may take some time before they are reflected in outcomes. Thus longer than 2 years follow up may be required before dissection complications appear. Moreover, the higher short-term complication rate of TEVAR compared to medical care alone highlights an important observation that any procedure carries an inherent risk.

\section{Expanded TEVAR Indications: Predictors of Outcome in Uncomplicated Type B Aortic
Dissection Patients}

The long-term risk of developing aneurysm, rupture, or malperfusion still exists despite effective medical therapy of aortic dissection. On the other hand, some patients are successfully managed for many years with medical treatment alone. Thus, further classification of the uncomplicated aortic dissection group into high risk and well- controlled populations may allow surgeons to intervene earlier and ultimately improve long-term outcomes. In the last several years, a 
number of new anatomical and medical criteria have been associated with long-term prognosis

\section{Anatomical factors}

Those with large entry tear diameters $(>10 \mathrm{~mm})$ are at increased risk of future complication, hypothesized to be a result of persistent high false lumen pressures [11]. Even further, some have suggested that the cumulative size of entry tears is what influences false lumen hemodynamics [13]. In addition, a larger false to true lumen ratio of diameters may be associated with worse long-term outcomes. This ratio can give insight into to the propagation of fluid traveling through each lumen and thus have implications such as malperfusion and elevated aortic wall stress. Moreover, a large aortic diameter $(>3.5 \mathrm{~cm})$, at initial presentation, is associated with aneurysm progression and potentially, rupture

\section{Medical factors}

Rapid disease progression of an initially uncomplicated dissection can be an indication to intervene. Some patients may experience early complication symptoms (e.g. aortic dilatation, pain) at a faster rate, and thus TEVAR may help limit further severe complications. In addition, patient's presenting with persistent pain despite medical therapy can be appropriate candidates for earlier intervention if a surgeon feels that the potential benefits outweigh the risks. Lastly, those with a persistent hypertension resistant to medical treatment are also at elevated risk of disease progression [14].

\section{Our Experience}

When treating type B aortic dissection initially diagnosed as uncomplicated, surgeons need to risk stratify patients based on symptoms and likelihood of complications. Those who have anatomy suitable for TEVAR and are healthy enough to undergo endotracheal intubation and general anesthesia, still need to have significant life expectancy to support intervention. This is a crucial point, as the current data suggests that the effects of TEVAR take some time. Given that a patient is considered safe to operate and exhibits high-risk features discussed above, the healthcare center must have the technical capacity to perform such a procedure. Specifically, fixed fluoroscopic imaging and intravascular ultrasound are important tools for successful treatment.

Along with selecting the right patients, the optimal timing of intervention remains unclear. The INSTEAD trial showed acceptable outcomes when treating patients with TEVAR after 14 days from dissection [12]. If operated on too early, the aorta is more fragile and risk of retrograde dissection may be too high. If surgeons wait too long, the aortic septum may already be too stiff and the amount of potential remodeling may not outweigh the risk of the procedure. Thus, the subacute period, 2-6 weeks after dissection, seems to be the optimal time to operate. Of note, retrograde dissection is a potentially deadly complication of TEVAR. Since a large proportion of patients with type $B$ dissection have a dilated ascending aorta, they are at higher risk for retrograde dissection $[15,16]$. Thus any institution that plans on using TEVAR to treat uncomplicated dissection must have the ability to convert to open and have a cardiac surgeon available. Otherwise, the risk of intervening on these currently stable patients is too high.

\section{Conclusion}

In order to assure that early intervention with TEVAR is beneficial for uncomplicated type B dissections, a patientspecific approach should be implemented to identify those at elevated risk of poorer outcomes. Our recent review of the contemporary management of this population highlights early promising results of TEVAR and discusses a number of suggested protocols [17]. Ultimately, if a patient is to undergo prophylactic TEVAR, he or she must be at a center familiar with the technique and patient population, and have the technical capacity and infrastructure in place to address any surgical complication. Moreover, close long-term surveillance is needed to monitor disease progression and the potential need for re-intervention. As the use of TEVAR grows, larger studies comparing medication alone to early endovascular intervention will be crucial.

\section{References}

Wheat MW, Palmer RF, Bartley TD, Seelman RC (1965) Treatment of dissecting dneurysms of the aorta without surgery. J Thorac Cardiovasc Surg 50: 364-373.

Cooper M, Hicks C, Ratchford EV, Salameh MJ, Malas M (2016) Diagnosis and treatment of uncomplicated type B aortic dissection. Vasc Med.

Appoo JJ, Bozinovski J, Chu MW, El-Hamamsy I, Forbes TL, et al. ( 2016) Canadian Cardiovascular Society/Canadian Society of Cardiac Surgeons/Canadian Society for Vascular Surgery Joint Position Statement on Open and Endovascular Surgery for Thoracic Aortic Disease. Can J Cardiol 32: 703-713.

4. Fattori R, Cao P, De Rango P, Czerny M, Evangelista A, et al. (2013) Interdisciplinary expert consensus document on management of type B aortic dissection. J Am Coll Cardiol 61: 1661-1678.

Gysi J, Schaffner T, Mohacsi P, Aeschbacher B, Althaus U, et al. (1997) Early and late outcome of operated and non-operated acute dissection of the descending aorta. Eur J Cardiothorac Surg 11: 1163-1169.

6. Sueyoshi E, Sakamoto I, Hayashi K, Yamaguchi T, Imada T (2004) Growth rate of aortic diameter in patients with type B aortic dissection during the chronic phase. Circulation 110: 256-261.

7. Durham CA, Aranson NJ, Ergul EA, Wang LJ, Patel VI, et al. (2015) Aneurysmal degeneration of the thoracoabdominal aorta after medical management of type B aortic dissections. J Vasc Surg 62: 900-906.

8. Marui A, Mochizuki T, Mitsui N, Koyama T, Kimura F, et al. (1999) Toward the best treatment for uncomplicated patients with type $B$ acute aortic dissection: A consideration for sound surgical indication. Circulation 100: 275-280.

9. Kunishige $H$, Myojin $K$, Ishibashi $Y$, Ishii $K$, Kawasaki $M$, et al. (2006) Predictors of surgical indications for acute type $B$ aortic 
dissection based on enlargement of aortic diameter during the chronic phase. Jpn J Thorac Cardiovasc Surg 54: 477-482.

10. Kamman AV, Hector WL, Guido HW, Foeke JH, Heijmen RH, et al. (2016) Contemporary Management Strategies for Chronic Type B Aortic Dissections: A Systematic Review. PLoS One 11: e0154930.

11. Evangelista A, Salas A, Ribera A, Ferreira-González I, Cuellar H, et al. (2012) Long-term outcome of aortic dissection with patent false lumen: predictive role of entry tear size and location. Circulation 125: 3133-3141.

12. Nienaber CA, Kische S, Rousseau H, Eggebrecht H, Rehders TC, et al. (2013) Endovascular repair of type B aortic dissection: long-term results of the randomized investigation of stent grafts in aortic dissection trial. Circulation: Cardiovascular Interventions 6: 407-416.

13. Hughes GC (2015) Management of acute type B aortic dissection; ADSORB trial. J Thorac Cardiovasc Surg 149: S158 S162.
14. Trimarchi S, Eagle KA, Nienaber CA, Pyeritz RE, Jonker FH, et al. (2010) Importance of refractory pain and hypertension in acute type $B$ aortic dissection: insights from the International Registry of Acute Aortic Dissection (IRAD). Circulation 122: 1283-1289.

15. Williams JB, Andersen ND, Bhattacharya SD, Scheer E, Piccini JP, et al. (2012) Retrograde ascending aortic dissection as an early complication of thoracic endovascular aortic repair. J Vasc Surg 55: $1255-1262$.

16. Booher AM, Isselbacher EM, Nienaber CA, Froehlich JB, Trimarchi S, et al. (2011) Ascending thoracic aorta dimension and outcomes in acute type $B$ dissection (from the International Registry of Acute Aortic Dissection [IRAD]). Am J Cardiol 107: 315-320.

17. Bannazadeh M, Tadros RO, McKinsey J, Chander R, Marin ML, et al. (2016) Contemporary Management of Type B Aortic Dissection in the Endovascular Era. Surg Technol Int 28: 214-221. 